\title{
EVALUATION OF SIMVASTATIN EFFECTS ON GENE EXPRESSION OF CELL WALL PRECURSORS IN STAPHYLOCOCCUS AUREUS
}

\author{
Sofia P. A. de Lima*, Kátia P. Silva, Karina C. Müller
}

\begin{abstract}
Staphylococcus aureus infections are difficult to treat due to their ability to form biofilm and their resistance to antibiotics. Studies have shown that simvastatin has an antimicrobial effect against $S$. aureus, possibly due to the inhibition of the HMG-CoA reductase enzyme at early steps of the mevalonate pathway. This pathway generates important intermediates for the synthesis of peptideoglycans from prokaryotes cell wall. Therefore, the antimicrobial mechanism of action of statins is not understood. Thus, the present study investigated the antimicrobial activity and action of simvastatin on the mevalonate pathway and on cell wall precursors expression of S. aureus. Minimum Inhibitory Concentration (MIC) and time-kill assays with addition of mevalonate were determined for S. aureus ATCC 29213. qRT-PCR were carried out to evaluate the gene expression of uppP, uppS, murG and mraY under simvastatin exposure. MIC for simvastatin was from 31.25 to $15.62 \mu \mathrm{g} / \mathrm{mL}$. Mevalonate addition did not altered the bacteriostatic effect of simvastatin ( $p>0.05$, Two-way ANOVA). Gene expression of cell wall precurssors were not altered by $1 / 4$ MIC and 1/8 MIC simvastatin ( $p>0.05$, ANOVA). Therefore, qRT-PCR assays will be repeated to confirm those results. In conclusion, simvastatin may not inhibit mevalonate pathway and cell wall precursors formation. However, more studies need to be carried out to confirm these findings.
\end{abstract}

Key words: Staphylococcus aureus, Simvastatin, Gene Expression

\section{Introduction}

Statins are lipid-lowering drugs who act by inhibiting the HMG-CoA reductase enzyme in eukaryotic cells, inhibiting the production of mevalonate and resulting in a reduction in plasma levels of low-density lipoprotein (LDL-cholesterol) $)^{1}$.

Studies have shown that simvastatin exerts antimicrobial effect against several bacterial species, including $S$. aureus ${ }^{2,3}$. However, the mechanism of action of statins in bacteria is not yet understood. It is known that the mevalonate pathway generates intermediates, the isoprenoids, which acts as a lipid carrier essential for the synthesis of the cell wall in prokaryotes ${ }^{4}$.

Thus, the present study evaluated the effect of simvastatin (SIMV) on the expression of genes related to the enzymes involved in the formation of lipid I and lipid II (precursors of cell wall formation). Furthermore, it was verified whether the addition of mevalonate would alter the action of simvastatin in a time-kill assay.

\section{Results and Discussion}

The Minimum Inhibitory Concentration (MIC) of SIMV is in the range of 31.25 to $15.62 \mu \mathrm{g} / \mathrm{mL}$.

Time-kill experiments were performed in cell cultures of $S$. aureus exposed to MIC and $1 / 2$ MIC, with and without the addition of mevalonate $(2.5 \mathrm{mM})$, for $24 \mathrm{~h}$ (image 1). SIMV presented antimicrobial effect at MIC and $1 / 2 \mathrm{MIC}$, but mevalonate did not reverse the antimicrobial activity of SIMV (Image 1, p> 0.05, 2 way-ANOVA).

To evaluate the expression of the uppS, uppP, mraY, murG and 16S rRNA genes, $S$. aureus was exposed to concentrations of $1 / 4 \mathrm{MIC}$ and $1 / 8 \mathrm{MIC}$ of SIMV for $8 \mathrm{~h}$. The $2-^{\Delta \Delta C T}$ method was used for the relative quantification of gene expression. The results indicated that there was no significant alteration of the gene expression for the genes murG, uppP e uppS (Chart 1, p> 0.05, ANOVA).

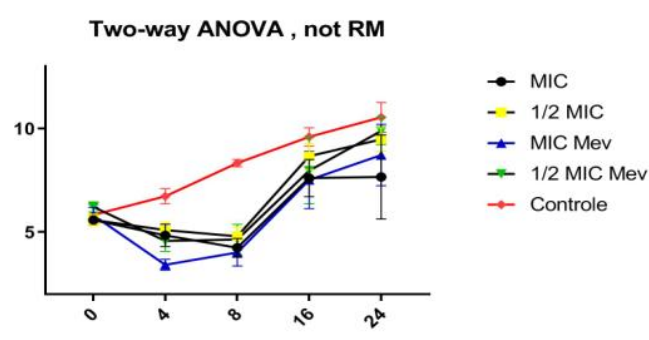

Image 1. Time-kill assay of S. aureus exposed to SIMV with and without Mevalonate for $24 \mathrm{~h}$.

Chart 1. Relative expression of murG, uppP and uppS under SIMV exposure (1/4 and $1 / 8 \mathrm{MIC})$ to control conditions.

\begin{tabular}{|c|c|c|c|c|c|}
\hline \multicolumn{2}{|c|}{ murG } & \multicolumn{2}{c|}{ uppP } & \multicolumn{2}{c|}{ uppS } \\
\hline $1 / 4 \mathrm{MIC}$ & $1 / 8 \mathrm{MIC}$ & $1 / 4 \mathrm{MIC}$ & $1 / 8 \mathrm{MIC}$ & $1 / 4 \mathrm{MIC}$ & $1 / 8 \mathrm{MIC}$ \\
\hline 0,5 & 1,13 & 0,6 & 0,52 & 1,16 & 0,62 \\
\hline 0,73 & 1,76 & 1,07 & 0,76 & 1,58 & 1,34 \\
\hline 0,89 & 0,85 & 1,99 & 1,19 & 1,50 & 0,9 \\
\hline
\end{tabular}

\section{Conclusions}

In conclusion, simvastatin may not inhibit mevalonate pathway and cell wall precursors formation. However, more studies need to be carried out to confirm these findings.

\section{Acknowledgement}

PIBIC - CNPq - bolsa de iniciação científica FAPESP - 2018/20593-0

\footnotetext{
${ }^{1}$ Endo, A. A gift from nature: the birth of the statins. Nature Medicine. 2008 Out;14(10):1050-52. doi:10.1038/nm1008-1050.

2 Jerwood S, Cohen J. Unexpected antimicrobial effect of statins. Journal Of Antimicrobial Chemotherapy. 2008:61(2):362-364. doi: 10.1093/jac/dkm496

${ }^{3}$ Bergman P, Linde C, Putsep K, Pohanka A, Normark S, Henriques-Normark $\mathrm{B}$, et al. Studies on the antibacterial effects of statins -in vitro and in vivo. PLoS One. 2011;6(8):e24394.

${ }^{4}$ Balibar CJ, Shen X, Tao J. The Mevalonate Pathway of Staphylococcus aureus . Journal of Bacteriology. 2009;191(3):851-861. doi:10.1128/JB.01357-08.
} 